\title{
Reconstrucción de las concentraciones de materia orgánica y nutrientes mediante espectrometría y análisis de diatomeas en tres embalses de Antioquia
}

\author{
Lina Juliana Gallo-Sánchez ${ }^{1 *}$, María Teresa Flórez-Molina ${ }^{1}$, Luis Norberto Parra-Sánchez² \\ ${ }^{1}$ Grupo de investigación en Modelación y Gestión Ambiental-GAIA, Facultad de Ingeniería, Universidad de Antioquia, Medellín, Colombia \\ ${ }^{2}$ Universidad Nacional de Colombia, Sede Medellín, Medellín, Colombia
}

\begin{abstract}
Resumen
Las técnicas de espectrometría y el análisis de las diatomeas permitieron excelentes ajustes para la estimación de la variación de las concentraciones de materia orgánica mediante el método de pérdida por ignición y la determinación de fósforo total y nitrógeno total Kjeldahl durante el tiempo de operación de los embalses de La Fe (37 años), Ríogrande II (21 años) y Porce II (9 años), localizados en Antioquia, Colombia. Los cambios en la paleo-geoquímica de los embalses no se acercaron al comportamiento esperado en los lagos, es decir, a una disminución exponencial en relación con la profundidad, lo cual se debe, en parte, a factores como la diagénesis tardía del sedimento y a la operación o manejo de los embalses. Sin embargo, las diatomeas presentes en el sedimento, así como la diversidad y el promedio anual del $\mathrm{pH}$ en la columna de agua, mostraron cambios significativos con respecto al llenado inicial del embalse y hasta el 2010 (año de recolección de los datos). En los embalses de La Fe y Ríogrande II se observó una disminución en la buena calidad inicial del agua, mientras que en Porce II estos indicadores mostraron una permanente eutrofización del sistema.
\end{abstract}

Palabras clave: materia orgánica, nitrógeno, fósforo, espectroscopia en el infrarrojo cercano, sedimentos.

Reconstruction of the concentrations of organic matter and nutrients using spectrometry and diatom analysis in three reservoirs in Antioquia, Colombia

\begin{abstract}
We present the results of a multiproxy reconstruction of the variation of organic matter using the loss-on-ignition method, and total phosphorus and Kjeldahl nitrogen during the operation of La Fe (37 years), Ríogrande II ( 21 years) and Porce II (9 years) reservoirs in Antioquia, Colombia, based on the analysis of diatoms and using nearinfrared spectroscopy. Changes in reservoir paleo-geochemistry did not approach the expected behavior for lakes, i.e., an exponential decrease with depth, due in part to factors such as a late diagenesis and the management of the reservoirs. However, diatoms present in the sediment, along with diversity and the annual average $\mathrm{pH}$ of the water column, showed significant changes from the initial filling of the reservoirs until 2010 (year in which the data were collected). In La Fe and Ríogrande II reservoirs, a decrease in water quality was observed, whereas in Porce II reservoir these indicators showed the continuing eutrophication of the system.
\end{abstract}

Key words: Organic matter, nitrogen, phosphorus, spectroscopy, near-infrared spectroscopy, sediment.

\section{Introducción}

Los sedimentos son compartimientos de almacenamiento de información de los ecosistemas acuáticos (Rosenberg, et al., 1998), incluidos los embalses, que ayudan a entender su historia, ya que pueden analizarse desde el punto de vista biológico (evaluando, por ejemplo, el polen, las crisofíceas y las diatomeas), así como físico y químico. De esta manera es posible determinar el papel de estos sistemas acuáticos como fuente o sumidero de nutrientes y contaminantes, estudiar la calidad del material degradado, o monitorizar su respuesta a la remediación o recuperación natural del estrés antropogénico (Malley, et al., 2000). Los sedimentos también influyen en el estado trófico del sistema y en la biodiversidad (Nürnberg, 1984; Søndergaard, et al., 2003), debido a que intercambian

\footnotetext{
*Correspondencia:

Lina Juliana Gallo Sánchez, julianaga2003@yahoo.com

Recibido: 26 de junio de 2014

Aceptado: 25 de noviembre de 2014
} 
iones con el cuerpo de agua. En comparación con muchos lagos naturales, los embalses acumulan sedimentos de forma rápida (Clark \& Wasson, 1986), lo que permite una mejor resolución en los estudios paleolimnológicos (Tibby, et al., 2010).

Las diatomeas (Bacillariophyceae) integran uno de los grupos biológicos más ampliamente usados en estudios paleolimnológicos (Stoermer \& Smol, 1999; Battarbee, et al., 2001) debido a su sensibilidad a los cambios en la calidad del agua, por lo que son buenos indicadores de las condiciones pasadas de $\mathrm{pH}$ (Battarbee, et al., 1999) y de las concentraciones de nutrientes (Hall \& Smol, 1999).

La producción, el aporte y la descomposición de la materia orgánica en el agua y en el sedimento influyen en la composición química de la columna de agua, en la distribución de fauna y flora y en las características geoquímicas del fondo, permitiendo así la comparación de la productividad en el embalse a lo largo del tiempo y del espacio. Los análisis de nitrógeno y fósforo se requieren para entender los principales ciclos de nutrientes, y en los estudios paleolimnológicos, para conocer tanto la perturbación antropogénica como la historia de la eutrofización (Malley, et al., 2000).

Los métodos tradicionales para la caracterización química de los sedimentos son laboriosos y costosos, por lo que su análisis llega a ser un factor limitante de la investigación y de los programas de monitorización ambiental. Además, la cantidad de muestras de núcleos de sedimento que se recupera es, en muchas ocasiones, insuficiente para los análisis físicos y químicos, en cuyo reemplazo, la técnica de espectroscopia de reflectancia en el infrarrojo cercano (near-infrared spectroscopy, NIRS), es un método de análisis químico rápido, alternativo o complementario, que no es destructivo ni contaminante (Malley, 1998). Esta tecnología ha sido ampliamente usada en la industria durante los últimos tres decenios para análisis cuantitativos y cualitativos (Batten, et al., 1995; Davies \& Williams, 1996), al tiempo que se ha demostrado su éxito en los análisis de carbono, nitrógeno y fósforo en sedimentos (Malley, et al., 1996, 1999; Rosén, et al., 2000 a 2000b, 2001, 2005), y metales pesados (Malley \& Williams, 1997). En Colombia solo se ha utilizado en la industria agrícola (Larrahondo \& Palau, 2001; Vásquez, et al., 2004; Vásquez \& Mayorga, 2005; Bastidas \& Carbonell, 2008; Botero, et al., 2009). La técnica de NIRS combina la espectroscopia, la estadística y la computación para generar modelos matemáticos que relacionan la composición química (presencia de grupos químicos activos) con los cambios de energía en la región correspondiente al rango infrarrojo cercano en tres pasos principales: calibración, validación y aplicación del modelo en muestras de concentración desconocida.

El objetivo de esta investigación fue establecer, a partir de núcleos de sedimento recuperados en cada uno de los embalses, los cambios en la concentración de la materia orgánica (LOI), el fósforo total y el nitrógeno desde el inicio del llenado hasta el 2010. Nuestro modelo de predicción se construyó con la ayuda de la técnica NIRS y del análisis de las diatomeas como organismos de control de dichas predicciones.

\section{Materiales y métodos}

Los embalses de La Fe, Ríogrande II y Porce II se localizan en la parte central del departamento de Antioquia (Figura 1) y sus principales características se presentan en la Tabla 1. En cada uno de los embalses se tomaron al azar 70 muestras de sedimento superficial con una draga Eckman, y se retiró la

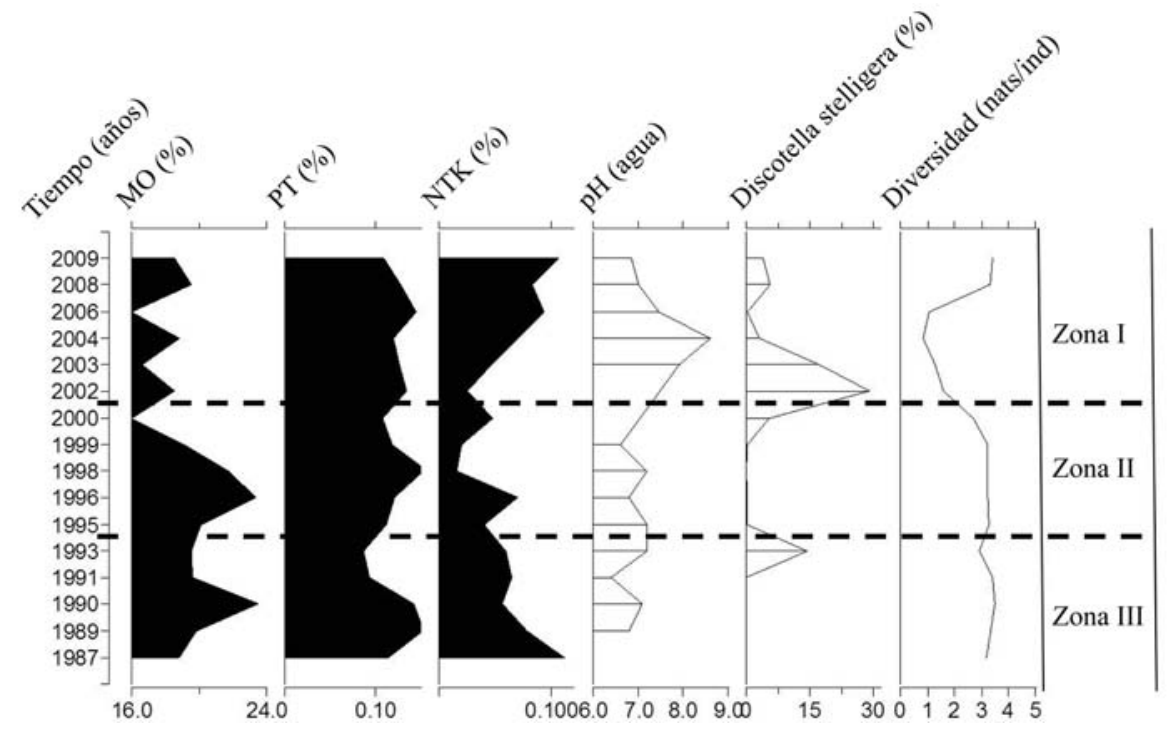

Figura 1. Ubicación de los embalses La Fe, Ríogrande II y Porce II y localización de los puntos de muestreo 
Tabla 1. Características principales de los embalses La Fe, Ríogrande II y Porce II

\begin{tabular}{|c|c|c|c|c|c|c|c|}
\hline \multirow[t]{2}{*}{ Embalse } & \multicolumn{2}{|c|}{ Ubicación } & \multirow{2}{*}{$\begin{array}{l}\text { Altura } \\
\text { m.s.n.m }\end{array}$} & \multirow{2}{*}{$\begin{array}{c}\text { Área } \\
\text { (ha) }\end{array}$} & \multirow{2}{*}{$\begin{array}{l}\text { Profundidad } \\
\text { máxima (m) }\end{array}$} & \multirow{2}{*}{$\begin{array}{l}\text { Llenado } \\
\text { (año) }\end{array}$} & \multirow[t]{2}{*}{ Uso } \\
\hline & Norte & Oeste & & & & & \\
\hline $\mathrm{LaFe}$ & $6^{\circ} 06^{\prime} 07^{\prime \prime}$ & $75^{\circ} 29^{\prime} 44^{\prime \prime}$ & 2.255 & $173 \mathrm{Km}^{2}$ & $20-25$ & $\begin{array}{l}\text { 1973,cuenco norte; } \\
\text { 1987, cuenco sur }\end{array}$ & $\begin{array}{l}\text { Abastecimiento del sistema de acueducto } \\
\text { y recreación }\end{array}$ \\
\hline Ríogrande II & 6030’15”' & 75³2’9,3”' & 2.200 & 1.041 & 47,2 & 1991 & $\begin{array}{l}\text { Generación hidroeléctrica, mejoramiento } \\
\text { ambiental y abastecimiento del sistema } \\
\text { de acueducto }\end{array}$ \\
\hline Porce II & $6^{\circ} 44^{\prime} 57^{\prime \prime}$ & $75^{\circ} 09^{\prime} 14^{\prime \prime}$ & 870 & 10 & 96,5 & 2002 & Generación hidroeléctrica \\
\hline
\end{tabular}

capa más superficial del sedimento (primeros $3 \mathrm{~cm}$, aproximadamente). Además, en la zona profunda y lejana de la pluma de los sedimentos de los tributarios más importantes en cada embalse, se recuperó un núcleo de sedimento con la ayuda de un nucleador de gravedad UWITEC. Los núcleos fueron seccionados al centímetro y a cada muestra se le determinó su espectro de infrarrojo cercano (near-infrared, NIR).

Las muestras de sedimento superficial se homogeneizaron y cuartearon en el laboratorio para determinar las concentraciones de materia orgánica (MO), fósforo total (PT) y nitrógeno total Kjeldahl (NTK) mediante química convencional, y el material restante se dejó para el análisis espectrofotométrico. En las muestras superficiales, el contenido de MO se determinó mediante pérdidas por ignición (LOI) a $550{ }^{\circ} \mathrm{C}$ (Dean, 1974). El NTK se realizó en húmedo mediante digestión ácida, 4500 Norg-B (APHA-AWWA-WEF, 2005), y el PT se analizó en seco con el método colorimétrico del ácido ascórbico, 4500-P-B (APHA-AWWA-WEF, 2005).

Para las determinaciones espectrofotométricas, las muestras de sedimento se llevaron al horno a $105^{\circ} \mathrm{C}$ durante 24 horas, al cabo de las cuales se trituraron una a una en un mortero eléctrico de ágata hasta obtener polvo fino. Posteriormente, el polvillo se colocó sobre un porta-muestras de cuarzo para su análisis en un espectrofotómetro marca Buchi (modelo NIR Master 500 con programa de adquisición Nirware 1.4), con el cual se obtuvo la reflectancia espectral cada $2,5 \mathrm{~nm}$ en el rango entre 1.000 y 2.500 nm (Osborne \& Fearn, 1986).

Los espectros de las muestras del sedimento superficial se utilizaron para la calibración del modelo predictivo utilizando los espectros NIRS, y la concentración de MO, PT y NTK de las muestras se empleó como grupo de variables explicativas; el modelo se obtuvo mediante técnicas estadísticas de regresión de mínimos cuadrados parciales (partial least squares regression, PLSR) implementadas en el lenguaje $\mathrm{R}$ (CRAN, 2009). Los espectros obtenidos de las muestras de los núcleos se utilizaron para estimar las concentraciones de MO, PT y NTK por medio del modelo predictivo. Las diatomeas y los cambios históricos en el pH se emplearon para contrastar de forma independiente los valores estimados.
Las diatomeas, elegidas con base en el perfil de nitrógeno estimado (nutriente limitante en los tres sistemas), se contaron en diferentes profundidades de los núcleos. Para dicha elección se estimó la media móvil y luego se seleccionaron los grupos con concentraciones similares; a partir de estos se eligió el número y las muestras a contar, teniendo en cuenta, además de la estratigrafía, que el número de muestras en cada grupo no fuera inferior al 30 \%. Para este análisis se trabajó con la especie de diatomea que mejor indicara el cambio del estado trófico.

Los datos sobre cambios históricos en el pH de las aguas de los embalses fueron suministrados por Empresas Públicas de Medellín y se comparó el promedio anual de los valores con las fechas equivalentes de los núcleos. Dado que los núcleos alcanzaron el fondo o basamento, y se conocía la fecha de inicio del llenado de los embalses, se pudo estimar la fecha del piso y del techo de cada núcleo. A los segmentos intermedios de un centímetro se les estimó una fecha con ayuda de la tasa lineal promedio de sedimentación, asumiendo que los posibles efectos de compactación y diagénesis hubieran sido constantes.

Además, debido a que en términos volumétricos el sedimento de los tres embalses es predominantemente mineral y con pocas variaciones en sus características sedimentológicas, se asumió que esto estaba relacionado con los aportes inorgánicos de la cuenca y que la aplicación de un modelo de sedimentación lineal para establecer la edad de la sucesion estratigráfica de los núcleos era razonable. Este presupuesto se respaldó por la adecuada coincidencia entre las fechas estimadas y las fechas reales de eventos históricos evidenciados en el sedimento, tales como los fenómenos de El Niño y La Niña. Los perfiles gráficos de $\mathrm{MO}$ y nutrientes, diatomeas y $\mathrm{pH}$ se obtuvieron en el programa C2 versión 1.7.2 (Juggins 2003-2011).

\section{Resultados}

La calibración del modelo predictivo se hizo mediante la regresión de los mínimos cuadrados parciales (PLSR), con la librería PLS y la función PLSR y el análisis de validación cruzada. Para establecer la precisión del modelo construido se aplicó el criterio de “dejar uno por fuera” (Leave One Out, 
LOO). Además, se evaluaron diferentes pre-tratamientos espectrales para establecer un modelo por variable con escaso número de componentes o variables latentes, un error bajo en la predicción de la validación cruzada (standard error of cross validation, SECV) y un coeficiente de determinación $\left(\mathrm{R}^{2}\right)$ lo más cercano posible a 1 (Botero, et al., 2009). De todos los pre-tratamientos, la dirección correcta de la señal (direct orthogonal signal correction, DOSC) fue el que cumplió con los requisitos mencionados, como se aprecia en la Tabla 2. Una vez se eligió el mejor modelo de predicción para cada variable y embalse, se calculó el estadístico RPD para evaluar la precisión y exactitud de las ecuaciones de predicción y constatar que fueran superiores a 3 (Williams \& Norris, 1987; Malley, et al., 2002).

Los modelos obtenidos para las variables analizadas en cada embalse mostraron que con solo dos variables latentes fue posible obtener un coeficiente de determinación superior al $80 \%$, valor que se considera aceptable para la estimación de las concentraciones de MO, PT y NTK en muestras de sedimento de concentración desconocida (Cozzolino, 2002; Malley, et al., 2002) (Tabla 2). Con base en la MO, el PT y el NTK estimados y en las diatomeas más representativas, los núcleos se dividieron en tres zonas de comportamientos distintos en cada uno de los embalses.

En el embalse La Fe, la zona I, que comprendía los años del 2001 al 2009, presentó una LOI que osciló entre 16,01 (2006) y 19,54 \% (2008), con una media de 18,02 \%, mientras que el PT varió entre 0,11 (2009) y 0,13 \% (2002 y 2003), con un valor medio de $0,13 \%$, en tanto que el NTK varió entre 0,03 (2002) y 0,11 \% (2009), con media de 0,07 \% (Figura 1). La diversidad varió entre 0,85 nats/ind (2004) y 3,40 nats/ ind (2009), con una media de 1,92 nats/ind. La diatomea

Tabla 2. Valores de los indicadores producto de la calibración y validación de las diferentes variables mediante la técnica NIRS en cada uno de los embalses

\begin{tabular}{lccccc}
\hline Embalse & Variable & $\begin{array}{c}\text { Número } \\
\text { de componentes }\end{array}$ & SECV & $\mathbf{R}^{2}$ & RPD \\
\hline \multirow{3}{*}{ La Fe } & MO & & 1,17 & 0,88 & 3,15 \\
& PT & & 65,55 & 0,96 & 4,93 \\
& NTK & & 73,66 & 0,97 & 5,40 \\
\hline \multirow{2}{*}{ Ríogrande II } & MO & & 1,01 & 0,96 & 3,00 \\
& PT & \multirow{2}{*}{2} & 126,4 & 0,93 & 5,76 \\
& NTK & & 196,6 & 0,96 & 5,99 \\
\multirow{2}{*}{ Porce II } & MO & & 0,63 & 0,87 & 3,48 \\
& PT & & 76,29 & 0,97 & 7,26 \\
\hline
\end{tabular}

MO: materia orgánica; PT: fósforo total; NTK: nitrógeno total Kjedahl; SECV: error estándar de validación cruzada; R2: coeficiente de determinación de la validación cruzada; RPD: desviación estándar de los datos de referencia (DS)/error estándar de la validación cruzada (SECV).
Central Discostella stelligera varió entre 0,22 (2006) y 29,11 \% (2002), con una media de 9,87 \%. El pH en la columna de agua presentó una media de 7,6 (Figura 2).

La zona II, que comprendía los años entre 1994 y 2001, presentó una LOI que varió entre 16,02 (2000) y 23,33 \% (1996), con una media de 20,07 \%, mientras que el PT varió entre 0,11 (1995 y 2000) y 0,15 \% (1998), con una media de 0,12, y el NTK osciló entre 0,02 (1999 y 1998) y $0,07 \%$ (1996), con un valor medio de 0,04 \% (Figura 1). La diversidad varió entre 2,71 nats/ind (2000) y 3,28 nats/ ind (1995), con una media de 3,11 nats/ind. D. stelligera presentó frecuencias entre 0 y 5,44 \% y una media de 1,24 \% (Figura 2). El pH en la columna de agua registró una media de 6,9 (Figura 2).

La zona III, que comprendía los años de 1987 (llenado del cuenco sur) a 1995, presentó valores de MO (estimados

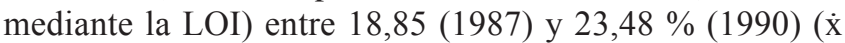
$=20,26 \%$ ). El PT fluctuó entre 0,09 (1991 y 1993) y $0,15 \%$ (1989), con un valor medio de 0,12 \%, mientras que el NTK osciló entre 0,06 (1990,1991 y 1993) y 0,11 \% (1987), con una media de 0,07 \% (Figura 1). La diversidad en esta zona osciló entre 2,93 nats/ind (1993) y 3,49 nats/ind (1990), con una media de 3,24 nats/ind. D. stelligera presentó frecuencias relativas de $0(1989,1990,1991)$ a $14,56 \%$ (1993) y una media de 2,93 \% (Figura 2). En esta zona el pH en la columna de agua registró una media de 6,88 (Figura 2).

Al descender estratigráficamente, la MO entre las zonas I y III registró un aumento, al igual que la diversidad y el pH, mientras que variables como el PT y la frecuencia relativa de $D$. stelligera disminuyeron. Por su parte, el NTK presentó en la zona intermedia una disminución conspicua en la concentración.

En el embalse Ríogrande II, la zona I, que comprendía los años entre 2001 y 2010, presentó valores de LOI que oscilaron entre 22,85 (2010) y 24,75 \% (2005), con una media de 23,97 \%, mientras que el PT varió entre 0,13 (2005 y 2010) y 0,25 \% (2003), con un valor medio de 0,17 \%. El NTK varió entre 0,01 (2008) y 0,28 \% (2001), con una media de $0,14 \%$ (Figura 3 ). La diversidad fluctuó entre 0,32 nats/ind (2003) y 1,30 nats/ind (2001), con una media de 0,83 nats/ind. La frecuencia relativa de Fragilaria familiaris varió entre 13,11 (2001) y 47,22 \% (2003), con una media de $32,80 \%$ (Figura 3 ). El pH medio en la columna de agua fue de 6,88 (Figura 3).

La zona II, que comprendía los años de 1993 a 2001, tuvo una LOI entre 22,40 (1999) y 23,84\% (1993) ( $\dot{\mathrm{x}}=22,40 \%)$, mientras que el PT varió entre 0,12 (1993) y 0,19 \% (1995 y 1999), con una media de $0,17 \%$, y el NTK entre 0,22 (1993) y $0,28 \%$ (1995) ( $\dot{x}=0,25 \%)($ Figura 2$)$. La diversidad varió entre 1,25 nats/ind (1999) y 3,06 nats/ind (1995), con una media de 2,27 nats/ind. La frecuencia relativa de F. familiaris 


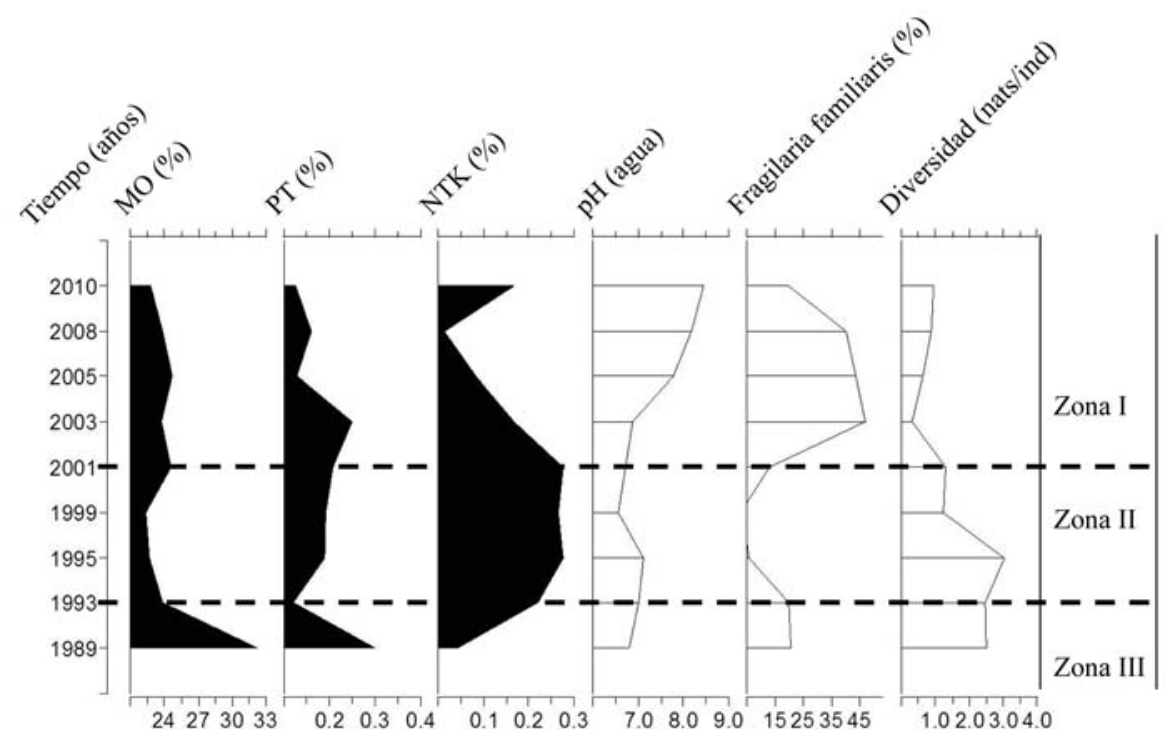

Figura 2. De izquierda a derecha, valores estimados de materia orgánica (MO), fósforo total (PT), nitrógeno total (NTK), frecuencia relativa (\%) de la diatomea Central Discostella stelligera y valores de diversidad (nats/ind) en el núcleo del embalse La Fe

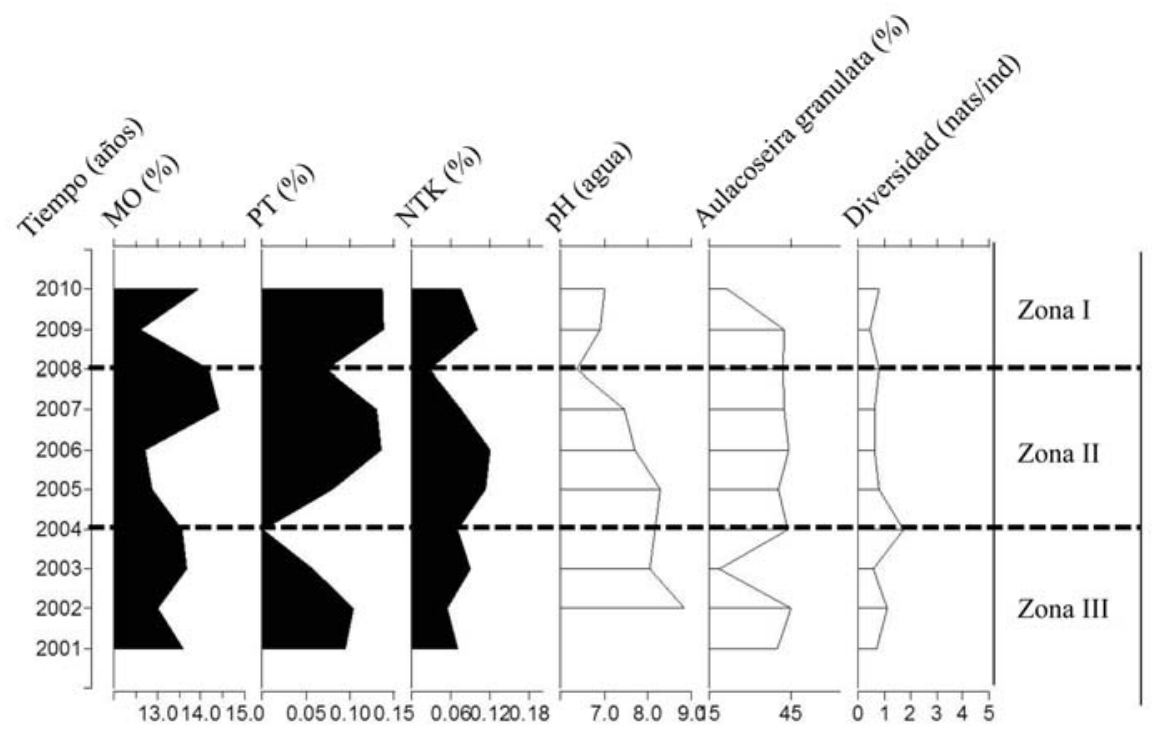

Figura 3. De izquierda a derecha, valores estimados de materia orgánica (MO), fósforo total (PT), nitrógeno total (NTK), frecuencia relativa (\%) de la diatomea Pennada Fragilaria familiaris y valores de diversidad (nats/ind) en el núcleo del embalse Ríogrande II

osciló entre 3,44 (1999) y 19,89 \% (1993), con una media de 9,78 \% (Figura 3). El pH medio en la columna de agua fue de 6,89 (Figura 3).

La zona III, que comprendía los años de 1989 a 1993, tuvo un valor medio de MO (estimado por LOI) de 32,21 \%, el de PT fue de $0,30 \%$ y el de NTK, de 0,04 \% (Figura 2). La diversidad fue de 2,51 nats/ind, mientras que la frecuencia relativa de $F$. familiaris fue de 20,89 \% (Figura 3). El valor medio del pH en la columna de agua fue de 6,80 (Figura 3).
Entre las zonas I y III del embalse Ríogrande II se encontró que, al igual que en el embalse La Fe, el PT y la diversidad aumentó, pero lo contrario ocurrió con el NTK, el pH en la columna de agua y la frecuencia relativa de F. familiaris.

En el embalse Porce II, la zona I, que comprendía los años de 2008 a 2010, presentó valores de MO (estimados mediante la LOI) entre 12,62 (2009) y 14,99\% (2010) ( $\dot{\mathrm{x}}=13,40 \%)$. El PT varió entre 0,09 (2008) y 0,14 \% (2008 y 2009), con una media de 0,13 \%. El NTK osciló entre 0,05 (2008) y 0,10 


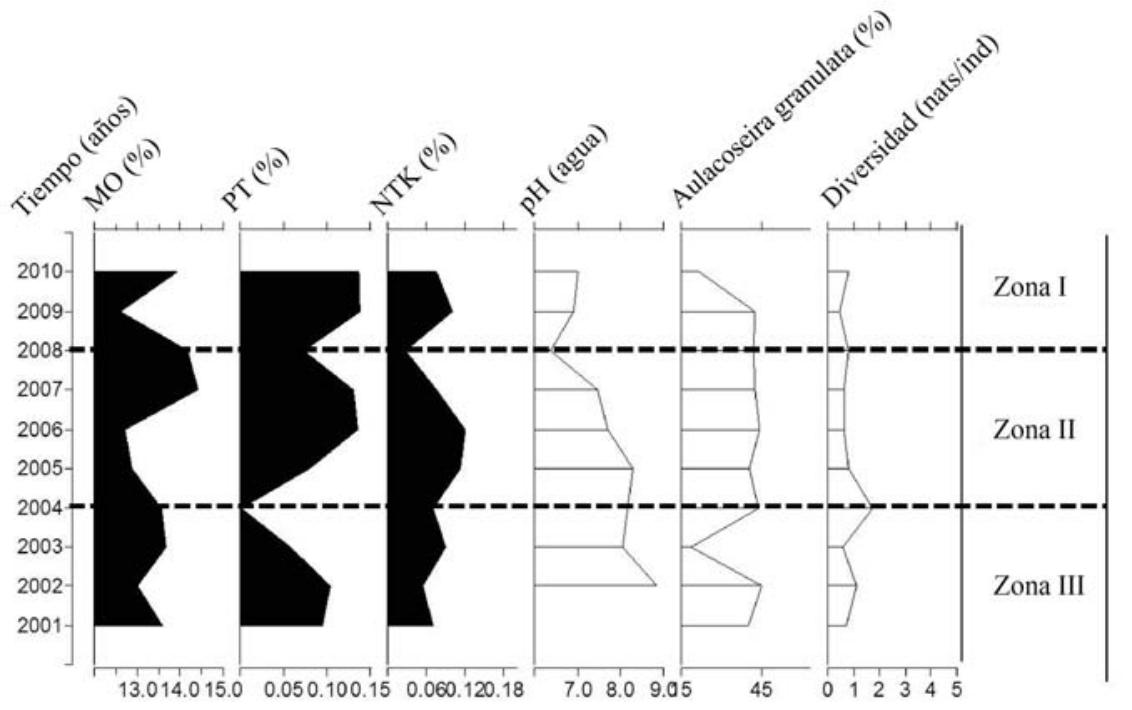

Figura 4. De izquierda a derecha, valores estimados de materia orgánica (MO), fósforo total (PT), nitrógeno total (NTK), frecuencia relativa (\%) de la diatomea Central Aulacoseira granulata y valores de diversidad (nats/ind) en el núcleo del embalse Porce II

(2009), con una media de 0,08 \% (Figura 4). La diversidad fue muy baja y varió entre 0,44 nats/ind (2008) y 1,03 nats/ ind (2009), con una media de 0,77 nats/ind, mientras que la frecuencia relativa de Aulacoseira granulata osciló entre 1,56 (2010) y 46,33 \% (2008), con una media de 34,16\% (Figura 3). El pH en la columna de agua registró un valor medio de 6,76 (Figura 4).

La zona II, que comprendía los años de 2004 a 2008, registró una LOI entre 12,30 (2005) y 15,63\% (2007; $\dot{x}=13,69)$, mientras que el PT varió entre 0,00 (2004) y 0,17 \% (2007) $(\dot{\mathrm{x}}=0,10 \%)$, y el NTK entre 0,01 (2008) y $0,13 \%$ (2005) $(\dot{\mathrm{x}}=0,10)$ (Figura 3$)$. La diversidad varió entre 0,43 nats/ ind (2005) y 1,73 nats/ind (2004), con una media de 0,77 nats/ind, mientras que la frecuencia relativa de $A$. granulata osciló entre 34,22 (2005) y 43,78 \% (2004), con una media de 42,60 \% (Figura 4). El pH en la columna de agua registró un valor medio de 7,82 (Figura 4).

La zona III, que comprendía los años de 2001 a 2004, tuvo una LOI que osciló entre 12,76 (2002) y 14,43 \% (2001), con una media de 13,47 \%. EL PT varió entre 0,06 (2001) y $0,13 \%$ (2002), con un valor medio de $0,09 \%$, mientras que el NTK varió entre 0,05 (2001) y 0,09 \% (2002 y 2003), con una media de $0,07 \%$. En relación con los descriptores de la comunidad a través del núcleo, se observaron valores altos de dominancia debidos principalmente a A. granulata. La diversidad varió entre 0,51 y 1,09 nats/ind en el año 2002, con una media de 0,77 nats/ind, mientras que la frecuencia relativa de $A$. granulata osciló entre 19,00 (2003) y 45,33 \% (2001), con una media de 36,08 \% (Figura 4). El $\mathrm{pH}$ en la columna de agua registró un valor medio de 8,44 (Figura 4).
Los datos mencionados muestran que al descender estratigráficamente, en el embalse Porce II los valores de PT y de NTK disminuyeron, mientras que los valores de $\mathrm{pH}$ en la columna de agua y la diversidad aumentaron. Por su parte, la frecuencia relativa de A. granulata y la LOI presentaron su pico máximo en la zona intermedia.

Al analizar las líneas de tiempo en los tres embalses, se encontró que en La Fe y Ríogrande II se registró una gran coincidencia con tres eventos de El Niño: 1994-1995, 1997-1998 y 2000-2003, y con dos eventos de La Niña: 1995-1996 y 19981999. En contraste, en el embalse Porce II solo se registró el último de los eventos de El Niño (Ideam, 2010). Los eventos de El Niño quedaron reflejados en los sedimentos como limos grumosos o fibrosos, finamente estratificados, con capas de entre 1 y $2 \mathrm{~cm}$ de espesor, de color pardo amarillento oscuro y pardo oscuro, probablemente relacionados con condiciones episódicas de reducción-oxidación, siendo los minerales y los silicofósiles los microcomponentes dominantes, mientras que durante los eventos de La Niña el sedimento se mostró limoso o limo arenoso, con un aumento conspicuo de partículas orgánicas (grumos, coágulos y resinas) y fragmentos minerales de $<0,03 \mathrm{~mm}$ de diámetro, lo que probablemente refleja una alta denudación en las cuencas que surten los embalses.

\section{Discusión}

Las ecuaciones de calibración indicaron que los modelos hallados pueden utilizarse para hacer predicciones confiables y precisas de MO (o LOI), PT y NTK en muestras de sedimento con una concentración desconocida. Utilizando la NIRS se han hallado resultados similares para estas variables en lagos de Norteamérica y Europa (Korsman, et al., 1999; Malley, et al., 1999, 2000; Birks, 2001, 2005). 
En lagos se esperaría que el perfil vertical de PT, NTK y MO exhibieran un decrecimiento exponencial con la profundidad, ya que algunas especies de fósforo, nitrógeno y carbono podrían, bajo condiciones naturales, decaer en el tiempo y, en consecuencia, generar concentraciones bajas en los sedimentos más profundos y antiguos (Machain \& Ruiz, 2006; Reitzel, et al., 2007; Trolle, et al., 2007). Un patrón análogo se esperaba para los embalses, pero este patrón no se encontró, posiblemente debido a factores como las condiciones de operación del sistema, los procesos diagenéticos y las variaciones en el potencial redox, que pueden producir cambios en el registro sedimentario y hace más difícil estimar el estado trófico solo con los análisis geoquímicos. Además, variables como la materia orgánica medida en sedimentos de lagos tropicales al parecer rara vez reflejan la productividad del sistema (Esteves,1998).

Sin embargo, para el caso del embalse La Fe, el aumento en la frecuencia relativa de la diatomea Central planctónica $D$. stelligera y la disminución de la diversidad de la comunidad de diatomeas al descender estratigráficamente, se han asociado con el enriquecimiento de nutrientes (Stoermer \& Kreis, 1978; Stoermer, et al., 1985; Morales \& Salazar, 2012). Esta inferencia se ve respaldada por la asociación de $D$. stelligera con ambientes eutróficos (Lowe, 1974; Taylor, et al., 2007).

Machain \& Ruiz (2006) sugieren que bajo condiciones de estrés ambiental la diversidad disminuye debido a que ocurre lo mismo con el número de especies tolerantes. Si la cantidad de alimento no es una limitante, estas tienden a desarrollarse en grandes cantidades debido a la falta de competencia. Además, el pH en la columna de agua también aumentó, lo que sugiere el deterioro en la calidad del agua. Este es el caso, por ejemplo, del lago Ambazari (Nagpur, Maharashstra), donde un $\mathrm{pH}>7$ se ha asociado con condiciones de eutrofia (Humane, et al., 2010). De aquí se infiere que entre los años 1985 y 2009, el embalse La Fe sufrió un proceso de eutrofización.

En el embalse Ríogrande II se observó que la diatomea Pennada F. familiaris, muy abundante hoy, un aumento en el perfil de sedimento desde el llenado, excepto para la zona II donde disminuye. No obstante, la diversidad de la comunidad de diatomeas disminuyó entre las zonas III y I, lo que indica que, al igual que en el embalse La Fe, se ha producido un cambio desfavorable de las condiciones ambientales del sistema. Además, $F$. familiaris se ha asociado a sistemas eutróficos (Carneiro, 2007), por lo que en la actualidad es un taxón dominante en el embalse. Como sucedió en el embalse La Fe, el valor medio anual del pH en la columna de agua aumentó entre las zonas III y I.

En el embalse Porce II, A. granulata, indicadora de ambientes eutróficos (Taylor, et al., 2007), ha dominado el sistema desde el inicio hasta la actualidad, como se pudo apreciar en el estudio; prueba de ello es la baja diversidad que se encontró en todo el registro sedimentario y que no ha variado mucho desde el llenado de embalse hasta hoy, indicando que en el sistema han prevalecido condiciones ambientales desfavorables debido a que el embalse recibe las aguas del río Medellín, el cual presenta una alta carga de contaminantes y nutrientes, pues recibe las aguas servidas tanto de los hogares como de las industrias del Valle de Aburrá.

\section{Conclusiones}

Utilizando la PLSR y el DOSC como pre-tratamientos, los modelos obtenidos en el presente estudio mediante la técnica NIRS para materia orgánica (LOI), fósforo total y nitrógeno total Kjeldahl en muestras de sedimento de los embalses objeto de estudio cumplieron las condiciones necesarias para emplearse en la estimación de tales compuestos en perfiles verticales de sedimento, lo cual no hubiese sido posible con los métodos convencionales, ya que las cantidades de muestras que usualmente se obtienen de los núcleos son muy pequeñas para ello.

La geoquímica por NIRS, las diatomeas y el $\mathrm{pH}$ permitieron, en conjunto, establecer las variaciones de la MO, el PT y el NTK de cada embalse desde el llenado hasta la época reciente. Se observó que los tres sistemas presentan en la actualidad condiciones de eutrofización.

\section{Agradecimientos}

Los autores agradecen a Empresas Públicas de Medellín; al doctor Jaime Palacio, coordinador del proyecto embalses, y al Posgrado de Biología de la Universidad de Antioquia; al doctor Orlando Ruíz, del laboratorio de suelos de la Universidad Nacional-Sede Medellín, y al doctor Kenneth Roy Cabrera.

\section{Referencias}

APHA-AWWA-WEF. 2005. Standard Methods for the Examination of Water and Wastewater. $21^{\text {st }}$ Ed. Maryland (USA): Port City Press.

Battarbee, R.W., Charles, D.F., Dixit, S.S., Renberg, I. 1999. Diatoms as indicators of surface water acidity. In E.F. Stoermer \& J.P. Smol (eds.). The Diatoms: Applications for the Environmental and Earth Sciences. (pp. 85-127). Cambridge, UK: Cambridge University Press.

Battarbee, R.W., Jones, V.J., Flower, R.J., Cameron, N.G., Bennion, H., Carvalho, L., Juggins, S. 2001. Diatoms Tracking Environmental Change Using Lake Sediments. Vol. 3. Terrestrial, Algal, and Siliceous Indicators. In J.P. Smol, H.J.B. Birks \& W.M. Last (eds.). (pp. 155-202). Dordrecht, the Netherlands: Kluwer Academic Publishers.

Bastidas, E. \& Carbonell, J.A. 2008. La espectroscopia en el infrarrojo cercano (NIR) para caracterizar el patrón espectral en los suelos. En Memorias del XIV Congreso 
Colombiano de la Ciencia del Suelo. Manejo del Suelo para la Mitigación del Cambio Climático. Sociedad Colombiana de la Ciencia del Suelo, Villavicencio (Colombia).

Batten, G.D., Flinn, P., Welsh, L.A., Blakeney, A. B. 1995. Leaping Ahead with Near-infrared Spectroscopy. In the Sixth International Conference on Near-infrared Spectroscopy, Lorne, Victoria, Australia.

Botero, J., Parra L.N. \& Roy, K. 2009. Determinación del nivel de nutrición foliar en banano por espectrometría de reflectancia. Revista de la Facultad Nacional de Agronomia. 62 (2): 5089-5098.

Carneiro, L.A. 2007. Fragilariophyceae (Ochrophyta) de águas doces do estado de São Paulo. (Tese Doutorado). Instituto de Botânica da Secretaria de Estado do Meio Ambiente. São Paulo, Brazil.

Clark, R.L. \& Wasson, R.J. 1986. Reservoir sediments. In P. DeDeckker \& Williams, W.D., (eds). Limnology in Australia. (pp. 497-507). CSIRO: Melbourne.

Cozzolino, D. (2002). Uso de la espectroscopia de reflectancia en el infrarrojo cercano (NIRS) en el análisis de alimentos para animales. Agrociencia. VI (2): 25-32.

Davies, A.M. \&. Williams, P. 1996. Near Infrared Spectroscopy: The Future Waves. In The Seventh International Conference on Near Infrared Spectroscopy. Chichester, West Sussex, UK.

Dean, W.E Jr. 1974. Determination of carbonate and organic matter in calcareous sediments and sedimentary rocks by loss on ignition: Comparison with other methods. Journal of Sedimentary Petrology. 44: 242-248.

Esteves, E. 1998. Fundamentos de Limnología. (II ed.). Finep, Río de Janeiro: Editorial Interciencia.

Hall, R.I. \& Smol, J.P. 1999. Diatoms as indicators of lake eutrophication. In E.F. Stoermer \& J.P. Smol (eds.). The Diatoms: Applications for the Environmental and Earth Sciences. Cambridge, UK: Cambridge University Press. pp. 128-168.

Humane, S.K., Bokade, P.R., Humane, S.S., Wankhade, A.S. 2010. Trophic status and ecological studies of the Ambazari Lake, Nagpur, Maharashstra, based on sedimentary diatoms. Current Science. 99 (6): 816-822.

Korsman, T., Nilsson, M.B., Landgren, K., Renberg, I. 1999. Spatial variability in surface sediment composition characterized by near-infrared (NIR) reflectance spectroscopy. Journal of Paleolimnology. 21: 61-71.

Larrahondo, J.E. \& Palau, F. 2001. Espectroscopia de infrarrojo cercano NIR. Principios generales [serie Procesos Industriales, $\left.N^{\circ} 1\right]$. Cali, Colombia: Cenicaña.

Lowe, R.L. 1974. Environment requirements and pollution tolerance of freshwater diatoms. Cincinnati, Ohio, USA: U.S.: Environmental Protection Agency.

Machain, M.L. \& Ruiz, C. 2006. Indicadores biofísicos en sedimentos acuáticos. En Especies, Espacios y Riesgos, cap. 13. I edición. México: Instituto Nacional de Ecología.
Malley, D.F., Williams P.C., Hauser, B.W., Hall, J. 1996. Prediction of organic carbon, nitrogen and phosphorus in freshwater sediments using near infrared reflectance spectroscopy. In A.M. Davies \& Williams, P. (eds). Near Infrared Spectroscopy. The Future Waves. Chichester, U.K: NIR Publications. pp. 691-699.

Malley, D.F. \& Williams, P.C. 1997. Use of near-infrared reflectance spectroscopy in prediction of heavy metals in freshwater sediment by their association with organic matter. Environmental Science of Technology. 31: 3461-3467.

Malley, D.F. 1998. Near-infrared spectroscopy as a potential method for routine sediment analysis to improve rapidity and efficiency. Water Science and Technology. 37: 181-188.

Malley, D.F., Rönicke, H., Findlay, D.L., Zippel, B. 1999. Feasibility of using near-infrared reflectance spectroscopy for the analysis of $\mathrm{C}, \mathrm{N}, \mathrm{P}$, and diatoms in lake sediments. Journal of Paleolimnology. 21: 295-306.

Malley, D.F., Lockhart, L., Wilkinson, P., Hauser, B. 2000. Determination of carbon, carbonate, nitrogen, and phosphorus in freshwater sediments by near-infrared reflectance spectroscopy: Rapid analysis and a check on conventional analytical methods. Journal of Paleolimnology. 24: 415-425.

Malley, D.F., Mcclure, C., Martin, P. D., Firlotte, N., Goldsborough, G., Sheppard, M. 2002. Evaluation of Near-infrared Spectroscopy as a Rapid Method for Estimating the Carbon Stored per Unit Area in a Wetland. Winnipeg: PDK Projects, Inc.

Morales, S. \& Salazar, M. 2012. Diatomeas perifíticas de lagos con diferente estado trófico en el departamento del Cauca (Colombia). Luna Azul. 35: 10-27.

Nürnberg, G.K. 1984. The prediction of internal phosphorus load in lakes with anoxic hypolimnia. Limnology and Oceanography. 29: 111-124.

Osborne, B.G. \& Fearn, F. 1986. Near Infrared Spectroscopy in Food Analysis. New York: John Wiley.

Reitzel, K., Ahlgren, J., DeBrabandere, H., Waldebäck, M., Gogoll, A., Tranvik, L., Rydin, E. 2007. Degradation rates of organic phosphorus in lake sediment. Biogeochemistry. 82: $15-28$.

Rosén, P., Dåbakk, E., Renberg, I., Nilsson, M., Hall, R. 2000. Near-infrared spectrometry (NIRS), a new tool to infer past climatic changes from lake sediments. The Holocene. 10: $161-66$.

Rosén, P., Hall, R., Korsman, T. \& Renberg, I. 2000. Diatom transfer-functions for quantifying past air temperature, $\mathrm{pH}$ and total organic carbon concentration from lakes in northern Sweden. Journal of Paleolimnology. 24: 109-23.

Rosén, P., Segerstro, U.L.F., Eriksson, L., Renberg, I, Birks, H.J.B. 2001. Holocene climatic change reconstructed from diatoms, chironomids, pollen and near-infrared spectroscopy at an alpine lake (Sjuodjijaure) in northern Sweden. The Holocene. 11 (5): 551-562. 
Rosén, P. 2005. Total organic carbon (TOC) of lake water during the Holocene inferred from lake sediments and near-infrared spectroscopy (NIRS) in eight lakes from northern Sweden. Biogeochemistry. 76: 503-516.

Rosenberg, D.M., Reynoldson T.B., Day, K.E., Resh, V.H. 1998. Role of abiotic factors in structuring benthic invertebrate communities in freshwater ecosystems. In C. G Ingersoll., T. Dillon \& G. R. Biddinger (eds.). Ecological Risk Assessment of Contaminated Sediments, chapter 10. FL, USA: SETAC Press, Pensacola. pp. 135-155.

Søndergaard, M., Jensen, J.P., \& Jeppesen, E. 2003. Role of sediment and internal loading of phosphorus in shallow lakes. Hydrobiologia. 506: 135-145.

Stoermer, E.F. \& Kreis, R.G Jr. 1978. Preliminary checklist of diatoms (Bacillariophyta) from the Laurentian Great Lakes. Journal Great Lakes Research. 4:149-169.

Stoermer, E.F., Wolin, J.A., Schelske, L., Conley, D.J. 1985. An assessment of ecological changes during the recent history of Lake Ontario based on siliceous microfossils preserved in the sediments. Journal of Phycology. 21: 257-27.

Stoermer, E.F. \& Smol, J.P. 1999. The Diatoms. Applications for the Environmental and Earth Sciences. Cambridge, UK: Cambridge University Press.
Taylor J.C., Harding, W.R., \& Archibald, G.M. 2007. An illustrated guide to some common diatom species from South Africa. WRC Report TT 282/07. Pretoria: Water Research Commission.

Trolle, D., Hamilton, D. P. \& Pilditch, C.A. 2010. Evaluating the influence of lake morphology, trophic status and diagenesis on geochemical profiles in lake sediments. Applied Geochemistry. 25: 621.632.

Tibby, J., Gell, P., Hancock, G. \& Clark, M. 2010. Complex reservoir sedimentation revealed by an unusual combination of sediment records, Kangaroo Creek Reservoir, South Australia. Journal of Paleolimnology. 43: 535-549.

Vásquez, D., Abadía, B. \& Arreaza, L.C. 2004. Aplicación de la espectroscopía de reflectancia en el infrarrojo cercano (NIRS) para la caracterización nutricional del pasto Guinea y del grano de maíz. Revista Corpoica. 5 (1): 49-55.

Vásquez, D. \& Mayorga, O.L. 2005. Uso de la espectroscopía de reflectancia en el infrarrojo cercano (Nirs) para la cuantificación rápida y precisa de triptófano en maíces. Revista Corpoica. 6 (1): 48-51.

Williams, P.C. \& Norris, K.H. 1987. Near-infrared Technology in the Agricultural and Food Industries. St. Paul, MN, USA: American Association of Cereal Chemists Inc. 


\title{
First report and significance of the staurolite metabasites associated to a sequence of calc-silicate rocks from the Silgará Formation at the central Santander Massif, Colombia
}

\author{
Carlos A. Ríos ${ }^{1, *}$, Oscar M. Castellanos ${ }^{2}$ \\ ${ }^{1}$ Grupo de Investigación en Geología Básica y Aplicada (GIGBA), Escuela de Geología, Universidad Industrial de Santander, Bucaramanga, Colombia \\ ${ }^{2}$ Grupo de Investigación en Geofísica y Geología (PANGEA), Programa de Geología, Universidad de Pamplona, Pamplona, Colombia
}

\begin{abstract}
The Silgará Formation metamorphic rocks have been affected by a Barrovian-type of metamorphism, which has occurred under medium-pressure and high-temperature conditions. Scarce intercalations of metabasites from millimeter up to centimeter scale occur in reaction bands observed in the gradational contact between garnet-bearing pelitic and calc-silicate rocks. In this study, we report for the first time the presence of staurolite metabasites in the Santander Massif (Colombian Andes), which is of particular interest since it is an unusual occurrence, taking into account that staurolite is most commonly regarded as an index mineral in metapelites and is not very well known from other bulk compositions and pressure and temperature conditions. Staurolite metabasites contain plagioclase, hornblende and staurolite, suggesting a history of prograde metamorphism up to amphibolite facies conditions. The origin of staurolite can be associated to aluminium-rich metabasites and, therefore, it is strongly affected by bulk rock chemistry. Taking into account mineral assemblages and geothermobarometric calculations in pelitic rocks, we suggest that the staurolite + hornblende association can be formed at least at 400 to $600{ }^{\circ} \mathrm{C}$ and $6 \mathrm{kbar}$ at the peak of prograde metamorphism. Retrograde reactions suggest that these rocks experienced nearly isobaric cooling accompanied by retrograde metamorphism.
\end{abstract}

Key words: Staurolite, amphibolite, Silgará Formation, metamorphism, central Santander Massif.

Primer reporte y significado de las metabasitas con presencia de estaurolita asociadas a una secuencia de rocas calcosilicatadas en la Formación Silgará de la región central del Macizo de Santander, Colombia

\begin{abstract}
Resumen
Las rocas metamórficas de la Formación Silgará fueron afectadas por un metamorfismo tipo barroviense en condiciones de presión media y alta temperatura. Las intercalaciones de anfibolitas en escala milimétrica a centimétrica son escasas en las bandas de reacción del contacto gradacional entre rocas pelíticas con granate y rocas calcosilicatadas. En el presente trabajo se reporta por primera vez la presencia de metabasitas con estaurolita en el Macizo de Santander (Andes colombianos), lo cual es de particular interés por lo inusual de su ocurrencia y porque la estaurolita comúnmente se considera más como un mineral índice en metapelitas y no se conoce muy bien a partir de otras rocas de diferente composición y condiciones de presión y temperatura. Las metabasitas con estaurolita contienen plagioclasa, hornblende y estaurolita, lo que sugiere una historia que abarca desde el metamorfismo prógrado hasta las condiciones de la facies anfibolita. El origen de la estaurolita puede asociarse a metabasitas ricas en aluminio, por lo cual está fuertemente afectada por el quimismo de la roca. Teniendo en cuenta las paragénesis minerales y los cálculos geotermobarométricos en rocas pelíticas, los autores proponen que la asociación estaurolita + hornblenda puede formarse al menos a $400-600{ }^{\circ} \mathrm{C}$ y 6 kbar en el pico de metamorfismo prógrado. Las reacciones retrógradas sugieren que estas rocas experimentaron un enfriamiento casi isobárico acompañado de metamorfismo retrógrado.
\end{abstract}

Palabras clave: estaurolita, anfibolita, Formación Silgará, metamorfismo, región central del Macizo de Santander.

\section{Introduction}

Staurolite occurs almost exclusively as a typical product of regional metamorphism in rocks of pelitic composition; however, it has been recorded as a rare constituent in metamorphic rocks of mafic composition (Selverstone, et a1., 1984). The occurrence of staurolite in metabasites has been reported by several authors: Miyashiro (1973), in metabasites of the Sambagawa metamorphic belt, Japan; Jan, et al. (1971), in amphibolite of the Timurgara ultramafic complex, Pakistan; 